\title{
Comparison of subfoveal choroidal thickness in eyes with CRVO and BRVO
}

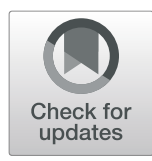

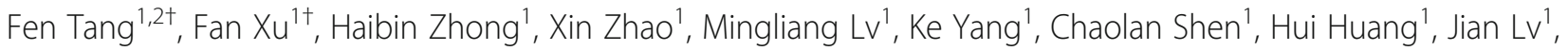
Siming Zeng ${ }^{1}$, Min Li ${ }^{1 *}$ and Qi Chen ${ }^{1 *}$

\begin{abstract}
Background: To evaluate the subfoveal choroidal thickness (SFCT) in eyes with macular edema (ME) secondary to retinal vein occlusion(RVO), and to investigate the short term response after a single intravitreal ranibizumab (IVR) injection. What is more, to compare SFCT and SFCT change between central RVO (CRVO) and branch RVO (BRVO).

Methods: In the retrospective study, we had collected 36-six treatment-naïve patients with unilateral ME secondary to RVO (including 19 CRVO and 17 BRVO). All patients had received IVR injection after newly diagnosed. The SFCT was measured at the onset and after 2 weeks of IVR injection. Paired $t$ test was performed to compare the SFCT of RVO eyes and fellow eyes, as well as the SFCT of pre-injection and post-injection. In further, independent $t$ test was used to compare SFCT and SFCT change between CRVO eyes and BRVO eyes.

Results: The mean SFCT at the onset was $326.03 \pm 30.86 \mu \mathrm{m}$ in CRVO eyes, which was significantly thicker than that in contralateral fellow eyes ( $p<0.01$, paired $t$ test), and reduced to $294.15 \pm 30.83 \mu \mathrm{m}$ rapidly after 2 weeks of IVR injection ( $p<0.01$, paired $t$ test). Similarly, the SFCT in BRVO eyes was significantly thicker than that in contralateral eyes at the onset, and decreased significantly after IVR injection. However, our findings showed that there was no statistically significant difference in SFCT and SFCT reduction after IVR injection between CRVO eyes and BRVO eyes.

Conclusions: The SFCT in eyes with ME secondary to CRVO and BRVO was significantly thicker than that in fellow eyes, and decreased significantly within a short time in response to a single IVR injection. In further, the study showed that SFCT and SFCT change had no correlation with RVO subtypes.
\end{abstract}

Keywords: Subfoveal choroidal thickness, Retinal vein occlusion, Macular edema, Ranibizumab

\section{Background}

Retinal vein occlusion (RVO) is a retinal vascular disorder characterized by obstruction of the retinal venous system, often associated with hypertension and coagulation abnormalities $[1,2]$. It is a common cause of visual handicap in the elderly throughout the world [3], and could be subdivided into central RVO (CRVO), branch RVO (BRVO) and hemi RVO (HRVO) according to the location of blockage [4]. Moreover, both CRVO and BRVO can be further classified into non-ischemic subtype and ischemic subtype based on the amount of retinal capillary perfusion [5]. Macular edema (ME) is one of the prominent complication in patients with ischemic RVO and can cause

\footnotetext{
* Correspondence: eyeminli@163.com; eyedoctorcq@163.com

${ }^{\dagger}$ Fen Tang and Fan Xu contributed equally to this work.

${ }^{1}$ Department of Ophthalmology, People's Hospital of Guangxi Zhuang

Autonomous Region, Nanning, Guangxi, China

Full list of author information is available at the end of the article
}

severe impairment of central vision [6]. Various treatment modalities had been used to treat ME, anti-vascular endothelial growth factor (VEGF) therapy had been demonstrated to be safe and effective among these available therapies [7-10].

The eyes with RVO may have abnormal choroidal vasculature, due to hydrostatic pressure and VEGF level [11]. Several studies had investigated subfoveal choroidal thickness (SFCT) in CRVO eyes and BRVO eyes, however, the results were contradictory. Some studies found that SFCT of affected RVO eyes had no significant difference compared with that of unaffected fellow eyes [11]. However, other studies showed that SFCT of RVO eyes was significantly thicker than that of unaffected fellow eyes [12] [13]. Besides, SFCT change after anti-VEGF therapy was also contradictory $[14,15]$. Most of the studies reported that the SFCT was decreased significantly after anti-VEGF

(c) The Author(s). 2019 Open Access This article is distributed under the terms of the Creative Commons Attribution 4.0 International License (http://creativecommons.org/licenses/by/4.0/), which permits unrestricted use, distribution, and 
treatment $[12,16]$, while a few studies reported that SFCT didn't decrease after anti-VEGF treatment [9]. Thus, these contradictory results warrant further investigation.

VEGF level was demonstrated as the principal factor which contribute to SFCT change [11]. Elevated VEGF expression could lead to increased capillary permeability and leakage in retina and choroid [2,17], is critically involved in the pathogenesis of ME secondary to $\operatorname{RVO}[18,19]$. Franco-Cardenas and colleagues found that ischemic index in CRVO was much higher than that in BRVO [20], what is more, Yasuda and colleagues found that aqueous VEGF concentration in CRVO eye was significantly higher than that in BRVO eye [21], these studies suggested that retinal ischemia in CRVO was more severe than that in BRVO. Therefore, we assume that SFCT of CRVO eye may be thicker than that of BRVO eye, however, it is uncertain and need to be demonstrated.

The present study was aimed to further investigate the SFCT in CRVO and BRVO eyes respectively, and to evaluate its short term response after a single IVR injection. More importantly, to compare SFCT and SFCT change after IVR injection between CRVO eye and BRVO eye.

\section{Methods}

In the retrospective case series, we collected and evaluated the data of 36 patients with unilateral ME secondary to RVO. Nineteen patients had CRVO, and 17 patients had BRVO. The diagnosis was determined according to the fundus examination and fluorescein angiography. Inclusion criteria were as the follows: (1) the age ranged from 50 to 70 years; (2) recent-onset (less than 1.5 months) and treatment-naïve when presented to the hospital; (3) was ischemic subtype and had received at least one intravitreal ranibizumab injection after newly diagnosed; (4) had follow-up of at least 2 weeks; (5) had comprehensive ophthalmic examinations before and after treatment. Patients were excluded if their fellow eyes had any macular disorder such as age-related degeneration (AMD), polypoidal choroidal vasculopathy (PCV) or central serous chorioretinopathy (CSC). Patients were also excluded if the affected eyes or fellow eyes had any of the following criteria: (1) axial length > $26.00 \mathrm{~mm}$ or $<22.00 \mathrm{~mm}$; (2) a history of pars plana vitrectomy or other intraocular surgeries within half year. The present study followed the tenets of the declaration of Helsinki and was approved by the ethics committee in hospital. The subjects had been informed written consent on the study.

Data collected from patients' medical records included age, axial length, gender, systemic diseases, and SFCT value at baseline and after 2 weeks of IVR injection. All patients had undergone standardized ophthalmic examinations, including best-corrected visual acuity (BCVA), intraocular pressure (IOP), slit-lamp biomicroscopy, funduscopic, fluorescein angiography (Heidelberg retina angiograph; Heidelberg Engineering Inc., Dossenheim, Germany), and enhanced depth imaging optical coherence tomography (EDIOCT) (Heidelberg Engineering Inc., Dossenheim, Germany) . BCVA was measured by the Early Treatment Diabetic Retinopathy Study (EDTRS). They had received intravitreal ranibizumab injection (Lucentis, $0.05 \mathrm{ml}, 0.5 \mathrm{mg}$ ) after newly diagnosed. After that, the following treatment strategies were varied based on clinically relevant benefits and risks, patients' anticipated visiting compliance, and the factor that whether the patients could afford the cost of ranibizumab. During the follow -up period, some of the patients were administered IVR injection monthly for three times, others received corticosteroids injection or laser photocoagulation. Nine patients with CRVO had received continuous IVR injection monthly for three times, whereas six patients with BRVO had received this treatment regimen. There were eight patients in both CRVO and BRVO groups who were administered corticosteroids injection or laser photocoagulation due to cost issue. Besides, five patients (including two CRVO and three BRVO) had lost to follow-up.

The demographic characteristics and SFCT value were collected. SFCT was measured from the outer border of the pigment epithelium to the choroidal scleral boundary, it was measured by 2 observers independently, and was recorded with the mean value. Statistical analysis was performed using Statistical Package for Social Science (SPSS) software (version 20.0, SPSS, Inc., Chicago, IL, USA). Continuous variables of the demographic characteristics were displayed as mean \pm standard deviation $(\mathrm{SD})$, categorical variables were displayed as the number of subjects and its percentage. Difference between continuous variables was analyzed by independent $t$ test, and Chi-square test was used for categorical variables. The SFCT values were displayed as mean \pm standard deviation (SD). The paired $t$-test was used to determine the difference in SFCT between RVO eye and its fellow eye. The SFCT between pre-injection and post-injection was also compared by paired $t$-test. The SFCT and SFCT change were compared between CRVO eye and BRVO eye by independent $t$-test. $P$ value $<0.05$ was considered statistically significant.

\section{Results}

Choroidal thickness was associated with the demographic characteristics of subjects. Axial length is an important predictor factor for the macular choroidal thickness, previous studies had demonstrated that the eyes with longer axial length would have thinner choroidal thickness [22, 23]. Age is another important factor, previous studies reported that choroidal thickness would decrease 10-15 $\mu \mathrm{m}$ [24] or $20-26 \mu \mathrm{m}[25,26]$ with age getting each 10 years older. As is summarized in Table 1 , no statistically difference was founded in axial length and age between CRVO 
Table 1 The patients' demographic characteristics

\begin{tabular}{|c|c|c|c|}
\hline & CRVO $(n=19)$ & $\mathrm{BRVO}(n=17)$ & $P$ \\
\hline Age, years (Mean $\pm S D$ ) & $57.37 \pm 9.75$ & $56.53 \pm 8.04$ & $N S^{a}$ \\
\hline Gender, Male (\%) & $12(63.16 \%)$ & $10(58.82 \%)$ & $N S^{b}$ \\
\hline Axial length, mm (Mean \pm SD) & $23.55 \pm 1.06$ & $23.92 \pm 1.03$ & $N S^{a}$ \\
\hline Ever smoker (n, \%) & $6(31.58 \%)$ & $8(47.06 \%)$ & $N S^{b}$ \\
\hline \multicolumn{3}{|l|}{ Systemic diseases } & $N S^{b}$ \\
\hline Hypertension (n, \%) & $11(57.89 \%)$ & $8(47.06 \%)$ & $N S^{b}$ \\
\hline Diabetes (n, \%) & $3(15.79 \%)$ & $4(23.53 \%)$ & $N S^{\mathrm{b}}$ \\
\hline Abnormal coagulation ( $\mathrm{n}, \%)$ & $4(21.05 \%)$ & $3(17.65 \%)$ & $N S^{b}$ \\
\hline BCVA(EDTRS letters) & $48.2 \pm 19.4$ & $50.2 \pm 11.3$ & $N S^{a}$ \\
\hline $\mathrm{IOP}(\mathrm{mmHg})$ & $14.2 \pm 2.5$ & $15.4 \pm 3.0$ & $N S^{a}$ \\
\hline
\end{tabular}

${ }^{\mathrm{a}}$ Independent $t$ test, ${ }^{\mathrm{b}}$ Chi-square test

group and BRVO group ( $p<0.05$, independent $t$ test), suggesting that the two groups were well balanced on axial length and age. Besides, there were no difference in gender distribution and smoking percentage between the two groups ( $p<0.05$, Chi-square test). The percentage of systemic diseases (such as hypertension, diabetes, and abnormal coagulation) had no statistically difference between the two groups. Additionally, the BCVA and IOP at baseline were shown in Table 1, no significant difference was found between CRVO and BRVO group.

Representative EDI-OCT images of CRVO are shown in Fig. 1a, b and c. Compared with unaffected fellow eyes, the SFCT of CRVO eyes was significantly thicker than that of fellow eyes, the mean SFCT of CRVO and fellow eyes were $326.03 \pm 30.86 \mu \mathrm{m}$ and $249.29 \pm 31.55 \mu \mathrm{m}$, respectively ( $p<0.001$, paired $t$ test). However, after 2 weeks of IVR injection, the mean SFCT of CRVO eyes reduced to $294.15 \pm 30.83 \mu \mathrm{m}$, which was significantly thinner than that before treatment (Fig. $1 \mathrm{~d}, p<0.001$, paired $t$ test).

Similarly, the SFCT of BRVO eyes was significantly thicker than that of fellow eyes, the mean SFCT of BRVO eyes and fellow eyes were $317.78 \pm 24.09 \mu \mathrm{m}$ and $255.21 \pm 20.40 \mu \mathrm{m}$, respectively (Fig. $2 \mathrm{~d}, p<0.001$, paired $t$ test). Moreover, the SFCT of BRVO eyes reduced to $287.65 \pm 24.42 \mu \mathrm{m}$ rapidly after IVR injection (Fig. 2e, $p<0.001$, paired $t$ test).

As was shown in Fig. 1 and Fig. 2, the SFCT in CRVO eyes and BRVO eyes showed a similar change trend, the thicker SFCT restored after IVR injection. In further, we compared SFCT and SFCT change between CRVO eyes and BRVO eyes. Unexpectedly, although the SFCT of CRVO eyes $(326.03 \mu \mathrm{m})$ was slightly thicker than that of BRVO eyes $(317.78 \mu \mathrm{m})$ at the onset, no significant difference was found between them (Fig. 3 a, $p>0.05$, independent $t$ test). The SFCT reduction after treatment were $31.88 \mu \mathrm{m}$ in CRVO eyes and $30.13 \mu \mathrm{m}$ in BRVO eyes, respectively. There was also no statistically significant difference in SFCT reduction between these two groups. (Fig. 3 b, C, p >0.05, independent $t$ test).

\section{Discussion}

The present study showed that the SFCT in eyes with ME secondary to RVO (including CRVO and BRVO) was significantly thicker than that in unaffected fellow eyes, and decreased rapidly within a short term in response to a single IVR injection, indicating that subfoveal choroid may be involved in the progress of $\mathrm{ME}$ secondary to RVO. The SFCT reduction after IVR was mainly caused by ranibizumab, which could permeate the retinal layer and extend to the choroid [27]. Furthermore, the SFCT and SFCT change were compared between CRVO group and BRVO group, no statistically significant difference was found, indicating that SFCT didn't have correlation with RVO subtype.

Macular choroidal thickness was correlated with disease severity and prognosis, EDI-OCT could provide a noninvasive method to evaluate the choroidal thickness in vivo $[28,29]$. Over the past several years, many studies had investigated the SFCT in macular-involved diseases. It was reported that the eyes with idiopathic macular hole [30] and dry AMD [31] had reduced SFCT, whereas the eyes with central serous chorioretinopathy (CSC) [32] and Vogt- Koyanagi-Harada (VKH) [33] had increased SFCT. ME is mainly caused by diabetic retinopathy and RVO. Previous studies showed that the SFCT in diabetic macular edema was thinner than that in normal eye [34], and was significantly correlated with the disease severity [35, 36]. With respect to ME secondary to RVO, there had been several studies to investigate choroidal thickness and the role of choroid in RVO eyes. As was mentioned above, Tsuiki and Coban Karatas found that the macular choroidal thickness in RVO eyes was thicker than that in unaffected fellow eyes [12, 13, 28]. In contrast, Du KF and colleagues reported that no significant difference was 


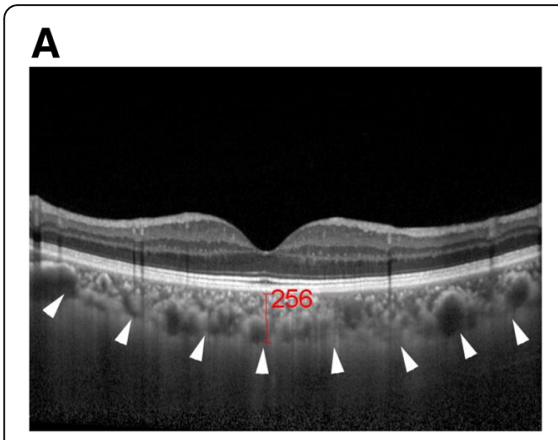

Fellow Eye
B

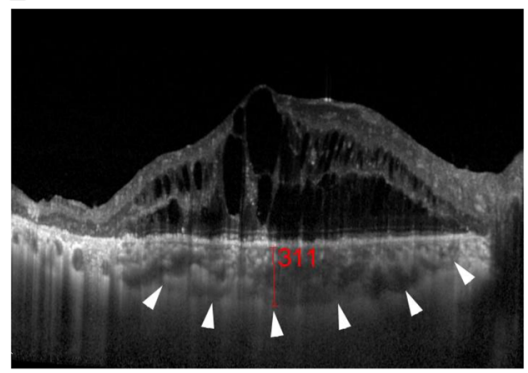

CRVO Eye (Before IVR injection)
C

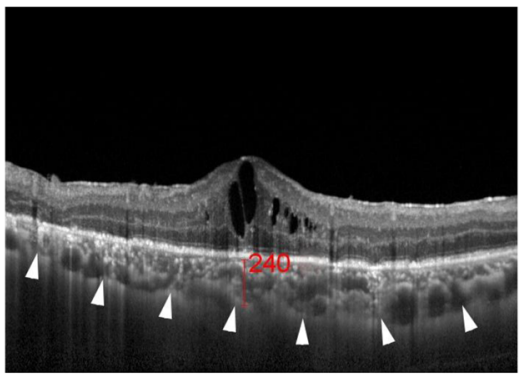

CRVO Eye (After IVR injection)
D

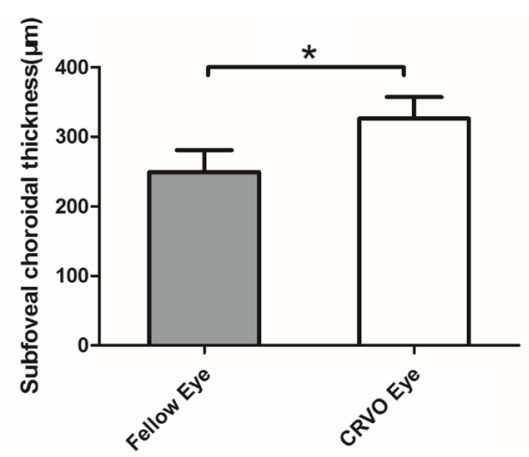

E

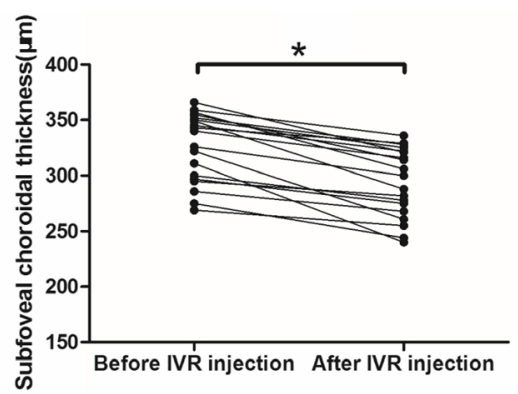

Fig. 1 Subfoveal choroidal thickness (SFCT) in patients with macular edema secondary to CRVO. a The representative EDI-OCT image of unaffected contralateral eye; $\mathbf{b}$ The representative EDI-OCT image of affected CRVO eye before IVR injection; c The representative EDI-OCT image of affected CRVO eye after IVR injection; White arrowheads point the choroid-scleral junction. SFCT was determined as the vertical distance from the hyperreflective line of the retinal pigment epithelium to the line of the choroid-scleral junction centered on the fovea, it was measured by 2 observers independently, and was recorded with the mean value. $\mathbf{d}$ Comparison of SFCT between the unaffected fellow eyes and CRVO eyes. e Comparison of SFCT in CRVO eyes between pre-IVR injection and post-IVR injection. The values were displayed as mean \pm standard deviation (SD). The paired $t$ test was used to evaluate the differences. ${ }^{*} p<0.05$ was considered statistically significant

found between RVO eyes and its fellow eyes [11]. One of the explanation for the conflicting result is the difference RVO phase, the subjects recruited by Tsuiki and Coban Karatas were at acute phase, while the study conducted by $\mathrm{Du}$ KF included the patients at longstanding and acute phase, the discrepancy between these studies may be contributed to the patients at longstanding phase. In our study, we collected the resent-onset and treatment-naivve patients, who were at acute phase, and our results were consistent with Tsuiki's findings. Furthermore, several studies demonstrated that choroidal thickness in RVO eyes decreased significantly following anti-VEGF treatment [12, 13, 16, 37], however, Park Jongyeop and colleagues reported that no SFCT change was found after anti-VEGF treatment [9]. The possible cause of this conflicting result might be the different follow-up period. Park Jongyeop evaluated SFCT after 12 months of treatment, while other studies evaluated it within a short follow-up period (ranged 1 month to 6 months). Our study evaluated SFCT after 2 weeks of IVR injection, which was a much shorter follow-up period. Our study still yield the similar finding with the studies which evaluated SFCT in the short term. The hypothesis is that SFCT may decrease in the short term after anti-VEGF treatment, and may restore in the long term. However, further investigation is needed to demonstrate it.

The initial choroidal thickness can be served as a biomarker of disease severity and a predictor of prognosis [35, 36, 38, 39]. Although there were several studies to evaluate the choroidal thickness in RVO, they focused on CRVO or BRVO separately [40,41]. It had been demonstrated that CRVO eyes had higher ischemic index and VEGF level compared with BRVO eyes. Moreover, increased VEGF would induce vascular hyperpermeability and dilated vessel in choroid layer, which is the main cause of increased choroidal thickness [42, 43]. Thus, it is supposed that the higher the VEGF level is, the thicker the choroidal thickness become. Therefore, we speculated that the SFCT of CRVO eyes might be thicker than that of BRVO eyes. However, our findings didn't show statistically significant difference between CRVO and BRVO. The possible reasons may be as follows:(1) The patients we collected were ischemic subtype, VEGF level in both CRVO and BRVO eyes was very high; (2) The sample size in each group was too small to detect a significant difference; (3) Besides VEGF, other unknown 


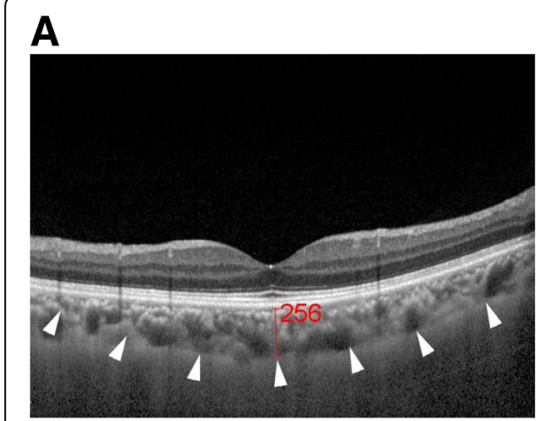

Fellow Eye

D

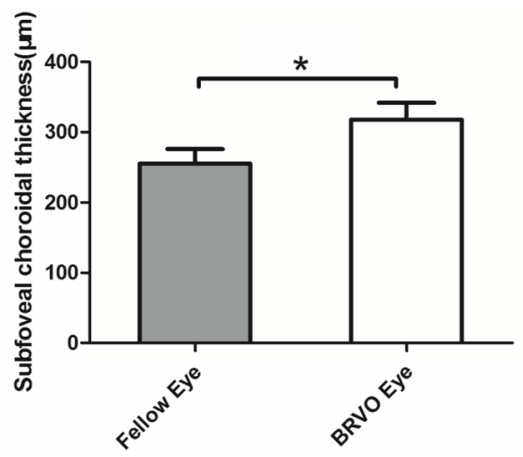

B

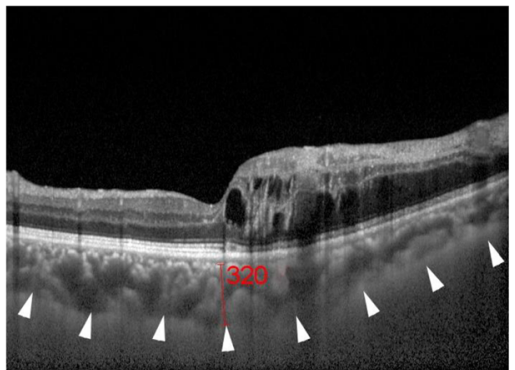

BRVO Eye (Before IVR injection)
C

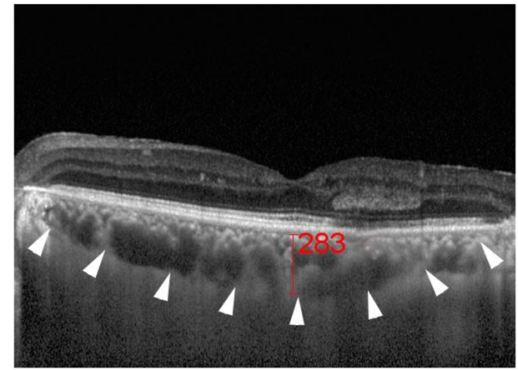

BRVO Eye (After IVR injection)

E

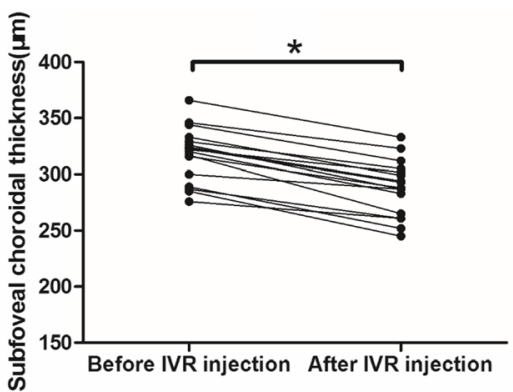

Fig. 2 Subfoveal choroidal thickness (SFCT) in patients with macular edema secondary to BRVO. a The representative EDI-OCT image of unaffected contralateral eye; $\mathbf{b}$ The representative EDI-OCT image of affected BRVO eye before IVR injection; $\mathbf{c}$ The representative EDI-OCT image of affected BRVO eye after IVR injection; White arrowheads point the choroid-scleral junction. SFCT was determined as the vertical distance from the hyperreflective line of the retinal pigment epithelium to the line of the choroid-scleral junction centered on the fovea, it was measured by 2 observers independently, and was recorded with the mean value. $\mathbf{d}$ Comparison of SFCT between the unaffected fellow eyes and BRVO eyes. $\mathbf{d}$ Comparison of SFCT in BRVO eyes between pre-IVR injection and post-IVR injection. The values were displayed as mean \pm standard deviation (SD). The paired $t$ test was used to evaluate the differences. ${ }^{*} p<0.05$ was considered statistically significant

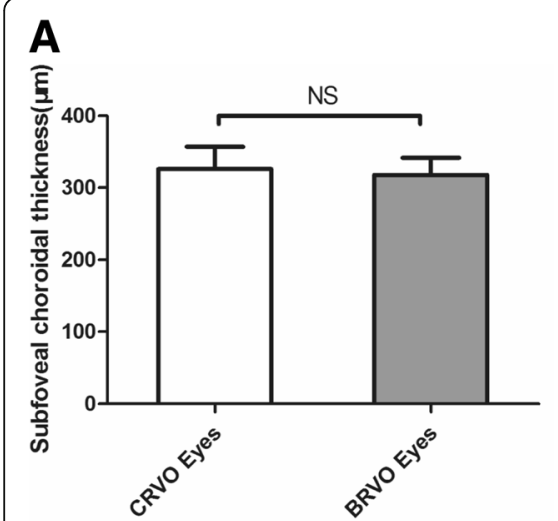

Before IVR injection

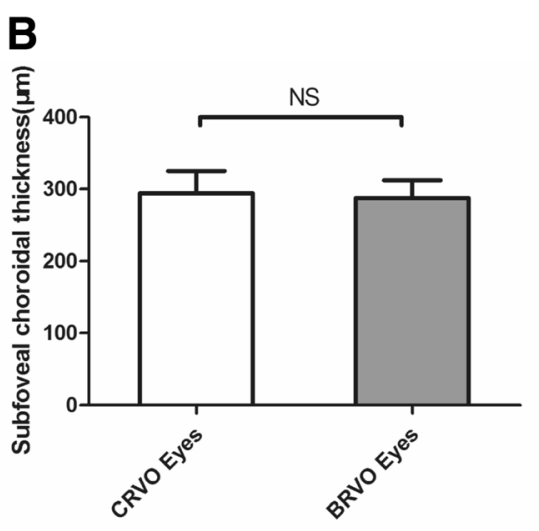

After IVR injection

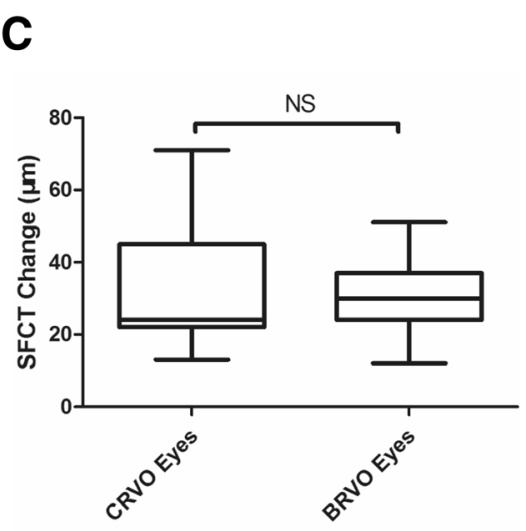

Fig. 3 There was no significant difference in SFCT and SFCT change between CRVO group and BRVO group. a Comparison of SFCT before IVR injection between CRVO group and BRVO group; b Comparison of SFCT after IVR injection between CRVO group and BRVO group; c Comparison of SFCT change between CRVO group and BRVO group. The independent $t$ test was used to evaluate the difference. $p>0.05$ was considered no significant difference (NS) 
factors might contribute to choroidal thickness change. Overall, the exact relationship between choroidal thickness and RVO severity require further investigation in the future study.

The present study had several limitations. First, the small sample size, short follow-up period and retrospectively designed study are the drawbacks. The prospective study with large number of subjects and long term follow-up is required in the future. Second, we only collected the patients with ME secondary to ischemic RVO, the patients included are not representative of the population of CRVO and BRVO, they only represent a small population of RVO patients who have the ME complication, this could be a selection bias. In order to further determine the exact relationship between SFCT and disease severity, the patients with non-ischemic RVO, the patients without ME and the patients with other complications (such as neovascularization and glaucoma) should be included in the future study. Third, choroidal thickness can present diurnal variation, the SFCT value is correlated with the measurement time, however, the EDI-OCT for all subjects was not performed within the same range in the study, this could be a possible bias. Fourth, a few subjects with diabetes had been included, although they did not have any sign of diabetic retinopathy according to comprehensive ophthalmic examinations, a potential bias might occur.

\section{Conclusion}

In conclusion, in recent-onset and treatment- naïve patients with ME secondary to RVO, the SFCT in affected eyes was statistically thicker than that in its unaffected contralateral eyes, and restored rapidly after 2 weeks of a single IVR injection. Our study may help to elucidate the conflicting results about the SFCT and SFCT change after anti-VEGF therapy. In further, our findings showed that there was no significant difference in SFCT and SFCT reduction between CRVO eyes and BRVO eyes, further study is still needed to investigate the exact relationship between SFCT and RVO severity.

\section{Abbreviations \\ AMD: Age-Related Degeneration; BRVO: Branch Retinal Vein Occlusion; CRVO: Central Retinal Vein Occlusion; CSC: Central Serous Chorioretinopathy; EDI-OCT: Enhanced Depth Imaging Optical Coherence Tomography; IVR: Intravitreal Ranibizumab; PCV: Polypoidal Choroidal Vasculopathy; RVO: Retinal Vein Occlusion; SFCT: Subfoveal Choroidal Thickness; VEGF: Vascular Endothelial Growth Factor}

\section{Acknowledgements}

Not applicable.

\section{Authors' contributions}

FT and FX performed the measurements. ML and QC were involved in planning and supervised the work. FT, HBZ and XZ processed the experimental data, performed the analysis, drafted the manuscript and designed the figures. MLL, KY, and CLS performed the ophthalmic examinations. $\mathrm{HH}, J \mathrm{~L}$ and SMZ collected the demographic characteristics and the choroidal thickness. All authors discussed the results and commented on the manuscript. All authors read and approved the final manuscript.

\section{Funding}

Supported by National Natural Science Foundation of China (No. 81560166, 81460087, 81660168, and 81760178) and Key Technologies R \& D Program of Guangxi (No.1598012-17). The funder had no role in the study design, data collection, data analysis, decision to publish or preparation of the manuscript.

\section{Availability of data and materials}

The datasets used and analyzed during the current study are available from the corresponding author on reasonable request.

\section{Ethics approval and consent to participate}

The study was approved by the Ethics Committee of the People's Hospital of Guangxi Zhuang Autonomous Region. The subjects had been informed written consent on the study.

\section{Consent for publication}

Not applicable.

\section{Competing interests}

The authors declare that they have no competing interests.

\section{Author details}

${ }^{1}$ Department of Ophthalmology, People's Hospital of Guangxi Zhuang Autonomous Region, Nanning, Guangxi, China. ²Department of

Ophthalmology, Renmin Hospital of Wuhan University, Wuhan, Hubei, China.

Received: 29 January 2019 Accepted: 12 June 2019

Published online: 21 June 2019

\section{References}

1. Feltgen $N$, Pielen A. Retinal vein occlusion : epidemiology, classification and clinical findings. Ophthalmologe. 2015;112(7):607-18 quiz 19-20.

2. Ip M, Hendrick A. Retinal vein occlusion review. Asia Pac J Ophthalmol (Phila). 2018;7(1):40-5.

3. Rogers S, McIntosh RL, Cheung N, Lim L, Wang JJ, Mitchell P, et al. The prevalence of retinal vein occlusion: pooled data from population studies from the United States, Europe, Asia, and Australia. Ophthalmology. 2010; 117(2):313-9 e1

4. Pulido JS, Flaxel CJ, Adelman RA, Hyman L, Folk JC, Olsen TW. Retinal vein occlusions preferred practice pattern((R)) guidelines. Ophthalmology. 2016; 123(1):P182-208.

5. Huang P, Niu W, Ni Z, Wang R, Sun X. A meta-analysis of anti-vascular endothelial growth factor remedy for macular edema secondary to central retinal vein occlusion. PLoS One. 2013;8(12):e82454.

6. Noma H, Funatsu H, Mimura T, Harino S, Hori S. Vitreous levels of interleukin-6 and vascular endothelial growth factor in macular edema with central retinal vein occlusion. Ophthalmology. 2009;116(1):87-93.

7. Jumper JM, Dugel PU, Chen S, Blinder KJ, Walt JG. Anti-VEGF treatment of macular edema associated with retinal vein occlusion: patterns of use and effectiveness in clinical practice (ECHO study report 2). Clin Ophthalmol. 2018;12:621-9.

8. Freund KB, Korobelnik JF, Devenyi R, Framme C, Galic J, Herbert E, et al. Treat-And-Extend Regimens With Anti-Vegf Agents In Retinal Diseases: a literature review and consensus recommendations. Retina. 2015;35(8): 1489-506.

9. Park J, Lee S, Son Y. Effects of two different doses of intravitreal bevacizumab on subfoveal choroidal thickness and retinal vessel diameter in branch retinal vein occlusion. Int J Ophthalmol. 2016;9(7):999-1005.

10. Qian T, Zhao M, Xu X. Comparison between anti-VEGF therapy and corticosteroid or laser therapy for macular oedema secondary to retinal vein occlusion: a meta-analysis. J Clin Pharm Ther. 2017;42(5):519-29.

11. Du KF, Xu L, Shao L, Chen CX, Zhou JQ, Wang YX, et al. Subfoveal choroidal thickness in retinal vein occlusion. Ophthalmology. 2013;120(12):2749-50.

12. Tsuiki E, Suzuma K, Ueki R, Maekawa Y, Kitaoka T. Enhanced depth imaging optical coherence tomography of the choroid in central retinal vein occlusion. Am J Ophthalmol. 2013;156(3):543-7 e1.

13. Coban-Karatas M, Altan-Yaycioglu R, Ulas B, Sizmaz S, Canan H, Sariturk C. Choroidal thickness measurements with optical coherence tomography in branch retinal vein occlusion. Int J Ophthalmol. 2016;9(5):725-9. 
14. Lee EK, Han JM, Hyon JY, Yu HG. Changes in choroidal thickness after intravitreal dexamethasone implant injection in retinal vein occlusion. $\mathrm{Br}$ J Ophthalmol. 2015;99(11):1543-9.

15. Arifoglu HB, Duru N, Altunel O, Baskan B, Alabay B, Atas M. Short-term effects of intravitreal dexamethasone implant (OZURDEX(R)) on choroidal thickness in patients with naive branch retinal vein occlusion. Arq Bras Oftalmol. 2016;79(4):243-6.

16. Yumusak E, Ornek K, Dikel NH. Comparison of choroidal thickness changes following intravitreal dexamethasone, ranibizumab, and triamcinolone in eyes with retinal vein occlusion. Eur J Ophthalmol. 2016;26(6):627-32.

17. Noma H, Funatsu $H$, Yamasaki M, Tsukamoto $H$, Mimura $T$, Sone $T$, et al. Pathogenesis of macular edema with branch retinal vein occlusion and intraocular levels of vascular endothelial growth factor and interleukin-6. Am J Ophthalmol. 2005;140(2):256-61.

18. Miller JW, Le Couter J, Strauss EC, Ferrara N. Vascular endothelial growth factor a in intraocular vascular disease. Ophthalmology. 2013;120(1):106-14

19. lijima $\mathrm{H}$. Mechanisms of vision loss in eyes with macular edema associated with retinal vein occlusion. Jpn J Ophthalmol. 2018;62(3):265-73.

20. Franco-Cardenas V, Shah SU, Apap D, Joseph A, Heilweil G, Zutis K, et al. Assessment of ischemic index in retinal vascular diseases using ultra-widefield fluorescein angiography: single versus summarized image. Semin Ophthalmol. 2017:32(3):353-7.

21. Yasuda S, Kachi S, Ueno S, Ushida H, Piao CH, Kondo M, et al. Electroretinograms and level of aqueous vascular endothelial growth factor in eyes with hemicentral retinal vein occlusion or branch retinal vein occlusion. Jpn J Ophthalmol. 2014;58(3):232-6.

22. Flores-Moreno I, Lugo F, Duker JS, Ruiz-Moreno JM. The relationship between axial length and choroidal thickness in eyes with high myopia. Am J Ophthalmol. 2013;155(2):314-9 e1.

23. Woodman EC, Read SA, Collins MJ. Axial length and choroidal thickness changes accompanying prolonged accommodation in myopes and emmetropes. Vis Res. 2012;72:34-41.

24. Ruiz-Medrano J, Flores-Moreno I, Pena-Garcia P, Montero JA, Duker JS, RuizMoreno JM. Macular choroidal thickness profile in a healthy population measured by swept-source optical coherence tomography. Invest Ophthalmol Vis Sci. 2014;55(6):3532-42.

25. Fujiwara A, Shiragami C, Shirakata Y, Manabe S, Izumibata S, Shiraga F. Enhanced depth imaging spectral-domain optical coherence tomography of subfoveal choroidal thickness in normal Japanese eyes. Jpn J Ophthalmol. 2012:56(3):230-5.

26. Abdolrahimzadeh S, Parisi F, Scavella V, Recupero SM. Optical coherence tomography evidence on the correlation of choroidal thickness and age with vascularized retinal layers in Normal eyes. Retina. 2016;36(12):2329-38.

27. Gaudreault J, Fei D, Beyer JC, Ryan A, Rangell L, Shiu V, et al.

Pharmacokinetics and retinal distribution of ranibizumab, a humanized antibody fragment directed against VEGF-A, following intravitreal administration in rabbits. Retina. 2007;27(9):1260-6.

28. Rayess N, Rahimy E, Ying GS, Pefkianaki M, Franklin J, Regillo CD, et al. Baseline choroidal thickness as a short-term predictor of visual acuity improvement following antivascular endothelial growth factor therapy in branch retinal vein occlusion. Br J Ophthalmol. 2019;103(1):55-59.

29. Rayess N, Rahimy E, Ying GS, Bagheri N, Ho AC, Regillo CD, et al. Baseline choroidal thickness as a predictor for response to anti-vascular endothelial growth factor therapy in diabetic macular edema. Am J Ophthalmol. 2015; 159(1):85-91 e1-3.

30. Karkhaneh R, Nikbakht M, Bazvand F, Oskouei AK, Ghasemi H, Ghassemi F. Choroidal thickness in idiopathic macular hole. J Curr Ophthalmol. 2017;29(1):45-9

31. Lu L, Xu S, He F, Liu Y, Zhang Y, Wang J, et al. Assessment of choroidal microstructure and subfoveal thickness change in eyes with different stages of age-related macular degeneration. Medicine (Baltimore). 2016;95(10):e2967.

32. Chung YR, Kim JW, Kim SW, Lee K. Choroidal thickness in patients with central serous chorioretinopathy: assessment of Haller and Sattler layers. Retina. 2016;36(9):1652-7.

33. Maruko I, lida T, Sugano Y, Go S, Sekiryu T. Subfoveal choroidal thickness in papillitis type of vogt-koyanagi-harada disease and idiopathic optic neuritis. Retina. 2016;36(5):992-9.

34. Eliwa TF, Hegazy OS, Mahmoud SS, Almaamon T. Choroidal thickness change in patients with diabetic macular edema. Ophthalmic Surg Lasers Imaging Retina. 2017;48(12):970-7.
35. Abadia B, Sunen I, Calvo P, Bartol F, Verdes G, Ferreras A. Choroidal thickness measured using swept-source optical coherence tomography is reduced in patients with type 2 diabetes. PLoS One. 2018;13(2):e0191977.

36. Rewbury R, Want A, Varughese R, Chong V. Subfoveal choroidal thickness in patients with diabetic retinopathy and diabetic macular oedema. Eye (Lond). 2016;30(12):1568-72.

37. Kim KH, Lee DH, Lee JJ, Park SW, Byon IS, Lee JE. Regional choroidal thickness changes in branch retinal vein occlusion with macular edema. Ophthalmologica. 2015;234(2):109-18.

38. Rayess N, Rahimy E, Ying GS, Pefkianaki M, Franklin J, Regillo CD, et al. Baseline choroidal thickness as a short-term predictor of visual acuity improvement following antivascular endothelial growth factor therapy in branch retinal vein occlusion. Br J Ophthalmol. 2019;103(1):55-9.

39. Hasegawa T, Kawano T, Maruko I, Koizumi H, lida T. Clinical findings of eyes with macular edema associated with branch retinal vein occlusion refractory to Ranibizumab. Retina. 2018;38(7):1347-53.

40. Quinlan PM, Elman MJ, Bhatt AK, Mardesich P, Enger C. The natural course of central retinal vein occlusion. Am J Ophthalmol. 1990;110(2):118-23.

41. Minturn J, Brown GC. Progression of nonischemic central retinal vein obstruction to the ischemic variant. Ophthalmology. 1986;93(9):1158-62.

42. Maruko I, lida T, Sugano Y, Ojima A, Sekiryu T. Subfoveal choroidal thickness in fellow eyes of patients with central serous chorioretinopathy. Retina. 2011;31(8):1603-8

43. Maruko I, lida T, Sugano $Y$, Furuta M, Sekiryu T. One-year choroidal thickness results after photodynamic therapy for central serous chorioretinopathy. Retina. 2011;31(9):1921-7.

\section{Publisher's Note}

Springer Nature remains neutral with regard to jurisdictional claims in published maps and institutional affiliations.

Ready to submit your research? Choose BMC and benefit from:

- fast, convenient online submission

- thorough peer review by experienced researchers in your field

- rapid publication on acceptance

- support for research data, including large and complex data types

- gold Open Access which fosters wider collaboration and increased citations

- maximum visibility for your research: over $100 \mathrm{M}$ website views per year

At $\mathrm{BMC}$, research is always in progress.

Learn more biomedcentral.com/submission 